\title{
COMPLEMENTARY LIDSTONE INTERPOLATION ON SCATTERED DATA SETS
}

\author{
F.A. Costabile, F. Dell'Accio, and F. Di Tommaso \\ Dipartimento di Matematica \\ Università della Calabria \\ via P. Bucci 30A 87036 Rende (Cs), Italy \\ ditommaso@mat.unical.it
}

Recently we have introduced a new technique for combining classical bivariate Shepard operators with three point polynomial interpolation operators. This technique is based on the association, to each sample point $V$, of a triangle with a vertex in $V$ and other ones in a neighborhood of $V$, to minimize the error of the three point polynomial interpolant. The combination inherits both degree of exactness and interpolation conditions, at each node $V$, of the polynomial interpolant, so that in [1] we succeed to extend Lidstone interpolation to scattered data sets by combining Shepard operators with the three point Lidstone interpolation polynomial [2]. Complementary Lidstone interpolation was recently introduced in [3] and drawn on by Agarwal, Pinelas and Wong. Complementary Lidstone interpolation naturally complements Lidstone interpolation. In this talk we generalize Complementary Lidstone interpolation to the case of bivariate scattered sample points. Numerical results are provided.

\section{References}

[1] R. Caira, F. Dell'Accio, F. Di Tommaso, On the bivariate ShepardLidstone operators, J. Comput. Appl. Math., submitted (2011).

[2] F.A. Costabile, F. Dell'Accio, Lidstone approximation on the triangle, App. Num. Math. 52 (2005), pp. 339-361.

[3] F.A. Costabile, F. Dell'Accio, R. Luceri, Explicit polynomial expansions of regular real functions by means of even order Bernoulli polynomials and boundary values, J. Comput. Appl. Math. 176 (2005) 77-90. 